\title{
Upper limits for mass and radius of objects around Proxima Cen from SPHERE/VLT
}

D. Mesa, ${ }^{1 \star}$ A. Zurlo, ${ }^{2,3,4}$ J. Milli, ${ }^{5}$ R. Gratton, ${ }^{1}$ S. Desidera, ${ }^{1}$ M. Langlois,,${ }^{6,4}$

A. Vigan, ${ }^{4}$ M. Bonavita, ${ }^{7,1}$ J. Antichi, ${ }^{1,8}$ H. Avenhaus, ${ }^{3,9,10}$ A. Baruffolo, ${ }^{1}$

B. Biller, ${ }^{10,7}$ A. Boccaletti, ${ }^{11}$ P. Bruno, ${ }^{12}$ E. Cascone, ${ }^{13}$ G. Chauvin, ${ }^{14,15}$

R. U. Claudi, ${ }^{1}$ V. De Caprio, ${ }^{13}$ D. Fantinel, ${ }^{1}$ G. Farisato,${ }^{1}$ J. Girard, ${ }^{5}$ E. Giro, ${ }^{1}$

J. Hagelberg, ${ }^{14,15}$ S. Incorvaia, ${ }^{16}$ M. Janson, ${ }^{17}$ Q. Kral, ${ }^{18}$ E. Lagadec, ${ }^{19}$

A.-M. Lagrange, ${ }^{14,15}$ L. Lessio, ${ }^{1}$ M. Meyer,${ }^{9,20}$ S. Peretti, ${ }^{21}$ C. Perrot, ${ }^{11}$ B. Salasnich, ${ }^{1}$

J. Schlieder, ${ }^{22,10}$ H.-M. Schmid, ${ }^{9}$ S. Scuderi, ${ }^{12}$ E. Sissa, ${ }^{1,23}$ C. Thalmann ${ }^{9}$

and M. Turatto ${ }^{1}$

Affiliations are listed at the end of the paper

Accepted 2016 November 28. Received 2016 November 25; in original form 2016 October 18

\begin{abstract}
The recent discovery of an earth-like planet around Proxima Centauri has drawn much attention to this star and its environment. We performed a series of observations of Proxima Centauri using Spectro-Polarimetric High-contrast Exoplanet REsearch (SPHERE), the planet-finder instrument installed at the European Southern Observatory (ESO) Very Large Telescope (VLT) UT3, using its near-infrared modules, InfraRed Dual-band Imager and Spectrograph (IRDIS) and IFS. No planet was detected directly, but we set upper limits on the mass up to $7 \mathrm{au}$ by exploiting the AMES-COND models. Our IFS observations reveal that no planet more massive than $\sim 6-7 M_{\text {Jup }}$ can be present within 1 au. The dual-band imaging camera IRDIS also enables us to probe larger separations than other techniques such as radial velocity or astrometry. We obtained mass limits of the order of $4 M_{\text {Jup }}$ at separations of 2 au or larger, representing the most stringent mass limits at separations larger than 5 au available at the moment. We also made an attempt to estimate the radius of possible planets around Proxima using the reflected light. Since the residual noise for the observations is dominated by photon noise and thermal background, longer exposures in good observing conditions could improve the achievable contrast limit further.
\end{abstract}

Key words: instrumentation: spectrographs-methods: data analysis-techniques: imaging spectroscopy - stars: individual: Proxima Centauri-planetary systems.

\section{INTRODUCTION}

After the recent discovery of a terrestrial planet around the star Proxima Centauri (Anglada-Escudé et al. 2016), new interest arose in the nearest star system to the Sun. While this planet, which has a separation of just 0.05 au with a period of $11.2 \mathrm{~d}$ and a minimum mass of $1.3 \mathrm{M}_{\oplus}$, cannot be imaged with the current instrumentation aimed at detecting the emitted light from extrasolar planets, e.g. Gemini Planet Imager (GPI) (Macintosh et al. 2006) and SpectroPolarimetric High-contrast Exoplanet REsearch (SPHERE) (Beuzit et al. 2008), it would, however, be interesting to have information about further possible objects at larger separations to characterize the system fully. Exploiting direct imaging observations, it is

^E-mail: dino.mesa@oapd.inaf.it possible to put some constraints on the mass and radius of other objects in the Proxima system. Similar work has been done in the past, exploiting both the radial velocity (RV) technique (Endl \& Kürster 2008; Zechmeister, Kürster \& Endl 2009; Barnes et al. 2014) and astrometric measurements (Lurie et al. 2014), but never exploiting direct imaging techniques. We have observed Proxima repeatedly with SPHERE in the past months, with the aim of obtaining precise astrometry of a background star that is undergoing a microlensing event caused by the approach of Proxima (Sahu et al. 2014). This star is clearly visible even when it is not undergoing the microlensing effect. This will give us a unique opportunity to measure the star mass directly (Zurlo et al., in preparation). However, the same data can be exploited to put some constraints on the mass of possible objects around Proxima, after calculating the contrast obtained from these observations. 
Table 1. SPHERE observations of Proxima Cen. DIT represents the time of each exposure expressed in seconds, while nDIT represents the number of frames for each data cube of the data set.

\begin{tabular}{cccrrrr}
\hline \multicolumn{1}{c}{ Date } & Obs. mode & \multicolumn{1}{c}{ Coronagraph } & nDIT;DIT(s) IRDIS & nDIT;DIT(s) IFS & Rot.Ang. $\left({ }^{\circ}\right)$ & Seeing $(\operatorname{arcsec})$ \\
\hline $2015-03-30$ & IRDIFS & N_ALC_YJH_S & $3 \times 12 ; 16$ & $3 \times 12 ; 16$ & 3.12 & 0.93 \\
$2016-01-18$ & IRDIFS & N_ALC_YJH_S & $7 \times 40 ; 16$ & $7 \times 20 ; 32$ & 25.74 & 2.20 \\
$2016-02-17$ & IRDIFS & N_ALC_YJH_S & $11 \times 10 ; 16$ & $11 \times 5 ; 32$ & 13.52 & 1.86 \\
$2016-02-29$ & IRDIFS & N_ALC_YJH_S & $7 \times 30 ; 16$ & $7 \times 15 ; 32$ & 22.56 & 0.78 \\
$2016-03-27$ & IRDIFS & N_ALC_YJH_S & $5 \times 40 ; 16$ & $5 \times 25 ; 32$ & 25.69 & 2.08 \\
$2016-04-15$ & IRDIFS & N_ALC_YJH_S & $6 \times 40 ; 16$ & $6 \times 20 ; 32$ & 28.72 & 0.62 \\
\hline
\end{tabular}

\section{DATA AND DATA REDUCTION}

Proxima Cen was observed during six different nights as part of the Guaranteed Time Observations (GTO) programme of the SPHERE consortium. The observations are listed in Table 1. All the observations were performed in the IRDIFS mode, with the Integral Field Spectrograph (IFS) (Claudi et al. 2008) operating at a spectral resolution $R=50$ in the wavelength range between 0.95 and $1.35 \mu \mathrm{m}$, with a field of view (FOV) of $1.7 \times 1.7 \operatorname{arcsec}^{2}$ corresponding to a maximum projected separation from the star of $\sim 1$ au and InfraRed Dual-band Imager and Spectrograph (IRDIS) (Dohlen et al. 2008) operating in the $H$ band with the $\mathrm{H} 23$ filter pair (wavelength $\mathrm{H} 2=$ $1.587 \mu \mathrm{m}$; wavelength $\mathrm{H} 3=1.667 \mu \mathrm{m}$ : Vigan et al. 2010) with a circular FOV with a radius of $\sim 5$ arcsec, corresponding to a maximum projected separation of $\sim 7$ au.

For both IFS and IRDIS, the data reduction was partly performed using the pipeline of the SPHERE data centre hosted at OSUG/IPAG in Grenoble. IFS data reduction was performed using the procedure described by Zurlo et al. (2014) and Mesa et al. (2015) to create calibrated data cubes composed of 39 frames at different wavelengths, to which we applied the principal components analysis (PCA: e.g. Soummer, Pueyo \& Larkin 2012; Amara, Quanz \& Akeret 2015) to reduce the speckle noise. The self-subtraction was taken into account appropriately by injecting fake planets at different separations into the data. An alternative data reduction was performed using the approach described in Vigan et al. (2015), leading to consistent results. IRDIS data were reduced following the procedure described by Zurlo et al. (2016) and applying the PCA algorithm for the reduction of speckle noise. An alternative reduction was performed following the procedure by Gomez Gonzalez et al. (submitted), leading to a comparable contrast. For the entire data set, the contrast was calculated following the procedure described by
Mesa et al. (2015), corrected by taking into account the smallsample statistics as devised in Mawet et al. (2014).

\section{RESULTS}

Given the very low Galactic latitude of Proxima, several sources were visible in the IRDIS FOV. One example of the reduced images is shown in the left panel of Fig. 1. Background stars move rapidly in these images, due to the large parallax and proper motion of Proxima, so that they can be identified very easily. One single background source (the star undergoing the microlensing event) was visible in the IFS FOV for three observing nights and it is shown in the right panel of Fig. 1. However, all the detected sources are background stars not bound to Proxima, so that no reliable companion candidate is detected in the SPHERE images.

Given the quality of the atmospheric conditions with respect to the other nights (see Table 1), the data from the night of 2016 April 15 give the best contrast, as shown in Table 2. Exploiting the very good conditions of this night, we were able to obtain a very deep $5 \sigma$ contrast. As listed in Table 2, the contrast is better than $10^{-6}$ at a separation of 0.4 arcsec using IFS and just above $10^{-6}$ at the same separation using IRDIS. These values are in good agreement with what is expected when SPHERE is observing a very bright target (see e.g. Zurlo et al. 2014; Mesa et al. 2015) and similar to those obtained until now during SPHERE observations for targets with a similar magnitude (see e.g. Vigan et al. 2015). In Fig. 2, we display the contrast in magnitude versus the separation expressed in au for both instruments. We can obtain a contrast better than 15 magnitudes at projected separations larger than 0.5 au with IFS, while with IRDIS we obtain a contrast better than 14 magnitudes at the same separation as IFS and we obtain a contrast better than 17 magnitudes at separations larger than $2.5 \mathrm{au}$.
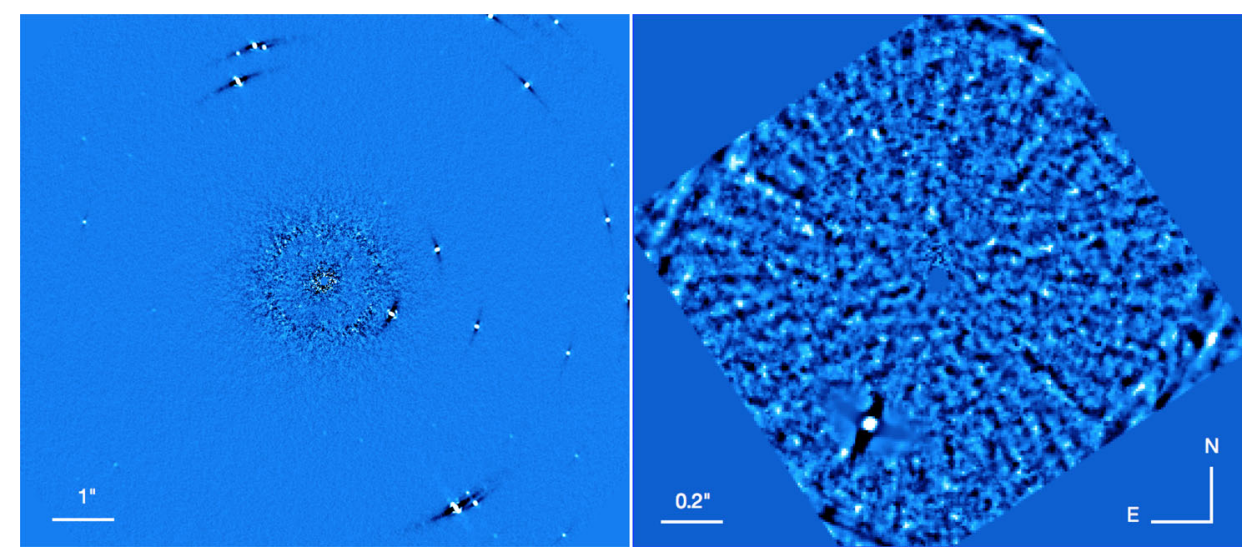

Figure 1. Final images obtained for IRDIS (left) and IFS (right). The IRDIS image is from the 2016 April 15 observation, while the IFS image is from the 2016 February 29 observation, to be able to show the background star that was no longer in the IFS FOV in April. 
Table 2. SPHERE IFS and IRDIS contrasts at a separation of 0.4 arcsec for the different observing nights.

\begin{tabular}{ccc}
\hline Date & $\begin{array}{r}\text { IFS contrast } \\
@ 0.4 \operatorname{arcsec}\end{array}$ & $\begin{array}{c}\text { IRDIS contrast } \\
@ 0.4 \operatorname{arcsec}\end{array}$ \\
\hline $2015-03-30$ & $8.01 \times 10^{-6}$ & $8.98 \times 10^{-5}$ \\
$2016-01-18$ & $5.55 \times 10^{-6}$ & $1.03 \times 10^{-5}$ \\
$2016-02-17$ & $3.82 \times 10^{-6}$ & $2.30 \times 10^{-5}$ \\
$2016-02-29$ & $1.79 \times 10^{-6}$ & $5.84 \times 10^{-6}$ \\
$2016-03-27$ & $3.83 \times 10^{-6}$ & $7.22 \times 10^{-6}$ \\
$2016-04-15$ & $8.58 \times 10^{-7}$ & $1.84 \times 10^{-6}$ \\
\hline
\end{tabular}

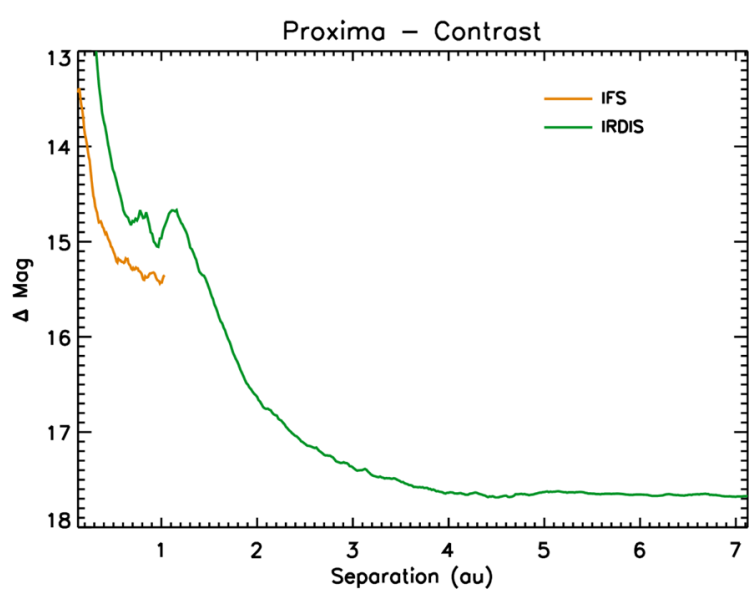

Figure 2. Magnitude contrast plot obtained for Proxima using SPHERE. The orange line represents the contrast using IFS, while the green line represents the contrast obtained using IRDIS.

Using the theorical model AMES-COND (Allard et al. 2003), we were able to set an upper limit on the mass of possible objects around Proxima. To this aim, we assumed a distance for Proxima of 1.295 pc (van Leeuwen 2007) and an age of $4.8 \pm 1$ Gyr (Thévenin et al. 2002; Bazot et al. 2016). Moreover, we assumed $J$ and $H$ magnitudes of 5.357 and 4.835 (Cutri et al. 2003), respectively, for the star. The upper mass-limit plots obtained in this way are displayed in Fig. 3 as solid lines. We found a mass limit of $\sim 7.5 M_{\text {Jup }}$ at a separation of 0.2 au and $\sim 6 M_{\text {Jup }}$ at separations larger than 0.6 au with IFS. On the other hand, using IRDIS we were able to obtain a limit of $8 M_{\text {Jup }}$ at $\sim 0.4$ au and lower than $5 M_{\text {Jup }}$ at separations larger than 2 au. Given the large uncertainties in the age of Proxima, we also calculated the mass limits considering ages of 3.8 and $5.8 \mathrm{Gyr}$, with the aim of showing how the mass limits change according to the stellar age and setting a more reliable range of mass limits. These results are shown as dashed lines in Fig. 3. In the same figure we included, as a comparison with our results, the mass limit obtained by Endl \& Kürster (2008) using the RV method (shown as red circles) and the limits obtained by Lurie et al. (2014) using astrometric measurements (blue squares).

While the April 2016 data clearly constitute the best data set that we obtained, we also combined the data from all observing epochs, attempting to increase the detection capability. This was performed using the procedure described in Vigan et al. (2015) and based on the MESS program (Bonavita et al. 2012), which is able to determine the probability of at least one detection during our observing dates calculated on a grid of values for the semi-major axis and companion mass. The results are shown in the left panel of Fig. 4. They are in good agreement with the results obtained in Fig. 3, but at shorter separations we are able to obtain better

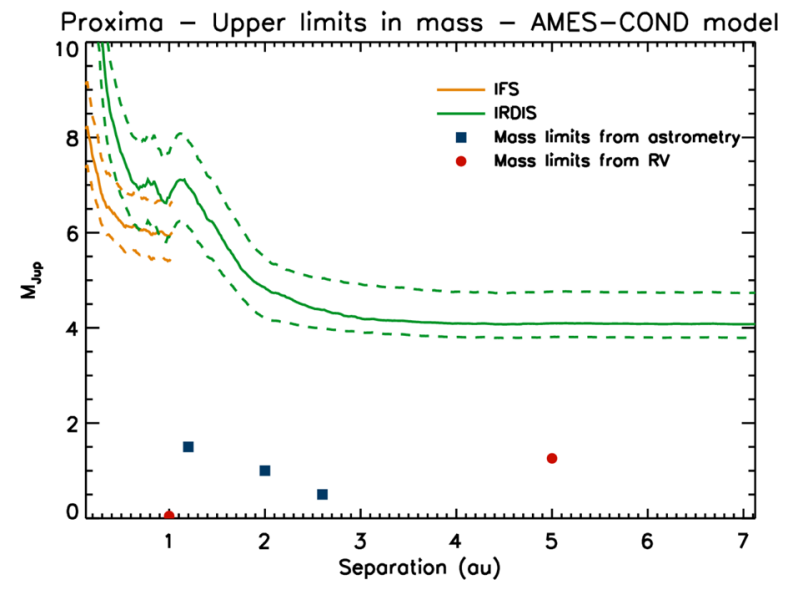

Figure 3. Mass limits for planets around Proxima calculated from the SPHERE contrast using the AMES-COND model. The orange lines represent limits from IFS, the green ones limits from IRDIS. The dashed lines are drawn to take into account the uncertainties in the stellar age. The limits from astrometry (blue squares) and from RV (red circles) are also shown, for comparison with our results.

sensitivity, as demonstrated by a comparison with the results of the same procedure performed using only the best epoch data displayed in the right panel of Fig. 4. This demonstrates, for example, that in this second case the 95 per cent probability of detection is cut at $0.4 \mathrm{au}$, while using all the observations combined we arrive at $0.2 \mathrm{au}$.

It is also possible to make an estimation of the limit in radius around Proxima, assuming planets are shining in reflected light.

However, the contribution to the luminosity of the planet in the regime around Proxima should be dominated mainly by intrinsic luminosity, while the contribution from reflected light should be less important. For this reason, the limits obtained through the reflected light are not very meaningful, with values ranging from $\sim 1.5 R_{\text {Jup }}$ at 0.2 au to $\sim 3 R_{\text {Jup }}$ at $\sim 1$ au and $\sim 10 R_{\text {Jup }}$ at $\sim 7$ au. Values of the radius of the order of $\sim 1 R_{\mathrm{Jup}}$, as foreseen from theoretical models, are then much more probable for substellar objects around Proxima.

\section{DISCUSSION AND CONCLUSIONS}

We presented the results of an analysis of the SPHERE data for Proxima Centauri. While it was not possible, as expected, to retrieve any signal from the planet recently discovered with the RV technique by Anglada-Escudé et al. (2016), we were able to set constraints on the mass and on the radius of other possible planets around this star.

Previous works put constraints on the minimum mass through the RV technique. One example is the value of $\sim 15 \mathrm{M}_{\oplus}$ at a separation of 1 au for the minimum mass $(M \sin i)$ given by Endl \& Kürster (2008). Other authors found similar results. The comparison of these limits with those obtained by direct imaging allows us to exclude face-on orbits for possible substellar objects. Extrapolating the results reported by Zechmeister et al. (2009) ${ }^{1}$ at larger separations, we can conclude that our results are consistent with those from RV at a separation of $\sim 7$ au, i.e. just at the limit of the IRDIS FOV.

Different results were obtained with astrometric measurements. For example, Lurie et al. (2014) set a mass limit of $\sim 1.5 M_{\text {Jup }}$ at

\footnotetext{
${ }^{1}$ The time span for these observations was around 7 years; for comparison, the foreseen orbital period for a planet orbiting at 7 au is $\sim 41$ years.
} 

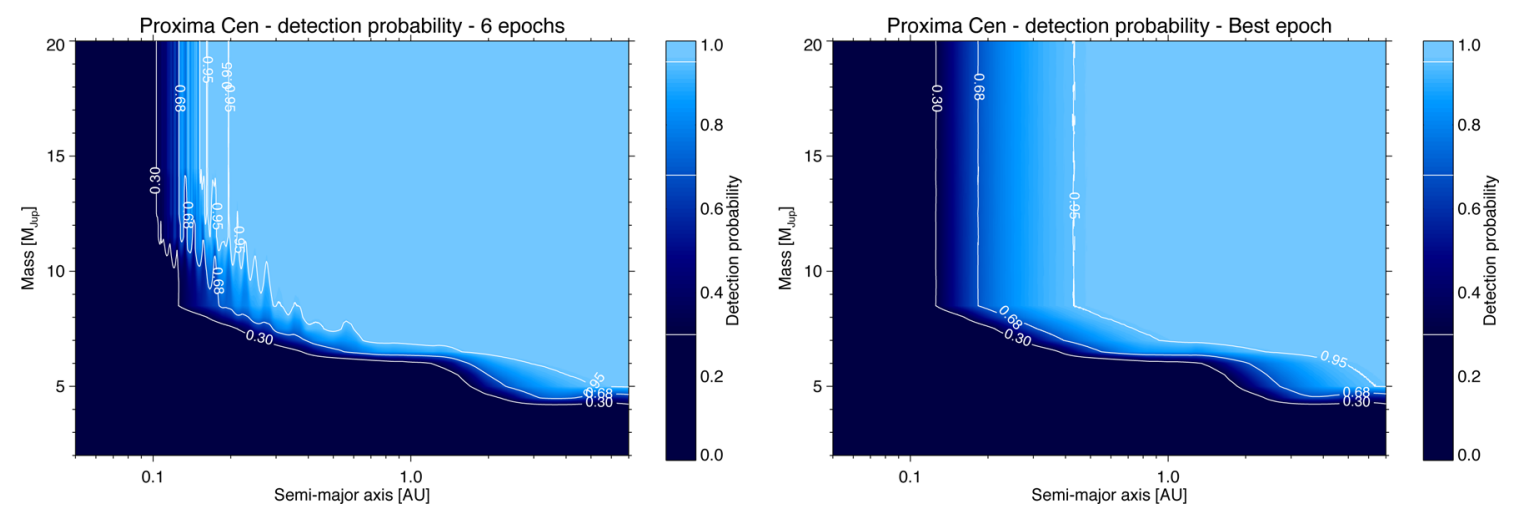

Figure 4. Left: mean probability of at least one detection of a substellar companion around Proxima Cen, using the combination of all the observations as a function of the companion mass and the semimajor axis. Right: the same, but using just best epoch data.

$1.2 \mathrm{au}, \sim 1 M_{\text {Jup }}$ at 2 au and $\sim 0.5 M_{\text {Jup }}$ at 2.6 au. These constraints are more sensitive to smaller planets than those we can obtain with SPHERE. Indeed, we obtain mass limits of $\sim 7.5 M_{\text {Jup }}$ at a separation of $0.2 \mathrm{au}$ and of the order of $6 M_{\mathrm{Jup}}$ at separations between 0.6 and $1 \mathrm{au}$. However, the wider IRDIS FOV allowed us to obtain mass limits at even larger separations, where RV and astrometry are less sensitive. We obtained mass limits better than $\sim 4 M_{\text {Jup }}$ at separations larger than $2 \mathrm{au}$. It is important to stress that these limits at separations larger than $\sim 5$ au concern a region unconstrained so far. Moreover, as pointed out by Dupuy, Liu \& Ireland (2011), modelbased substellar mass determinations could be overestimated. For this reason, the mass limits from direct imaging could be even lower than those determined with our measures.

We also attempted to obtain a limit for the radius using the reflected light. However, the limits that we obtained are not very stringent, probably because the intrinsic luminosity is more important than reflected light for objects around Proxima. Limits of $\sim 1 R_{\text {Jup }}$ foreseen through the theoretical models are probably more reliable for these substellar objects around Proxima.

We obtained these results with a total exposure time of $\sim 1 \mathrm{~h}$. Given that the residual noise from our observations is mainly dominated by photon noise at separations larger than 0.3 arcsec for IFS and 1.5 arcsec for IRDIS, we should be able to improve our contrast further with longer exposures taken in sky conditions comparable to those of 2016 April or better. Under these assumptions, we should be able to improve our contrast as the square root of the exposure time ratio. However, this improvement will not be comparable with the sensitivity reached by the other methods reported above. For example, a long exposure of $20 \mathrm{~h}$ taken during more than one night will enable us to reach a contrast of $1.89 \times 10^{-7}$ with IFS, corresponding to a mass limit of $4.9 M_{\text {Jup }}$ at a separation of $0.5 \operatorname{arcsec}$, still far from the limits obtained with RV and astrometry. To be able to improve the mass limit obtained with direct imaging further, we will have to wait for the availability of future instruments both in space (e.g. the James Webb Space Telescope: JWST) and from the ground, using future giant segmented mirror telescopes like the Giant Magellan Telescope (GMT: Johns 2008), Thirty Metre Telescope (TMT: Nelson \& Sanders 2008) and European Extremely Large Telescope (E-ELT: Gilmozzi \& Spyromilio 2007).

Using the online ETC for $J W S T,{ }^{2}$ we have calculated the contrast at different separations in the $L^{\prime}$ band for a $1 \mathrm{~h}$ observation and transformed it into mass limits, again using the AMES-COND models. We synthesized these results in Table 3, from which one can

\footnotetext{
${ }^{2}$ https://devjwstetc.stsci.edu/
}

Table 3. Contrast and mass limit for the $L^{\prime}$ band with JWST.

\begin{tabular}{lcc}
\hline $\begin{array}{c}\text { Separation } \\
(\operatorname{arcsec})\end{array}$ & $\begin{array}{c}\text { Magnitude } \\
\text { limits }\end{array}$ & $\begin{array}{c}\text { Mass limits } \\
\left(M_{\text {Jup }}\right)\end{array}$ \\
\hline 0.5 & 20.2 & 5.3 \\
1.0 & 21.7 & 3.5 \\
1.5 & 22.6 & 2.5 \\
2.0 & 23.6 & 1.7 \\
2.5 & 23.8 & 1.5 \\
\hline
\end{tabular}

see that we can have quite a good gain, especially at larger separations, where we can obtain a limit similar to those obtained through the RV.

\section{ACKNOWLEDGEMENTS}

Based on observations made with European Southern Observatory (ESO) telescopes at the Paranal Observatory in Chile, under programme IDs 095.D-0309(E), 096.C-0241(G), 096.D-0252(A), 096.C-0241(H), 096.C-0241(E) and 097.C-0865(A). We are grateful to the SPHERE team and all the people at Paranal for the great effort during the SPHERE early-GTO run. DM, AZ, RG, RUC, SD and ES acknowledge support from the 'Progetti Premiali' funding scheme of MIUR. We acknowledge support from the French ANR through the GUEPARD project grant ANR10-BLANC050401. QK acknowledges support from the EU through ERC grant number 279973. JH is supported by the GIPSE grant ANR-14CE33-0018. HA acknowledges financial support by FONDECYT, grant 3150643, and support from the Millennium Science Initiative (Chilean Ministry of Economy) through grant RC130007. SPHERE was funded by ESO, with additional contributions from CNRS (France), MPIA (Germany), INAF (Italy), FINES (Switzerland) and NOVA (Netherlands). SPHERE also received funding from the European Commission Sixth and Seventh Framework Programmes as part of the Optical Infrared Coordination Network for Astronomy (OPTICON) under grant number RII3-Ct-2004-001566 for FP6 (2004-2008), grant number 226604 for FP7 (2009-2012) and grant number 312430 for FP7 (2013-2016). Part of this has been carried out within the frame of the National Centre for Competence in Research Planets supported by the Swiss National Science Foundation. H.M.S. and M.R.M. acknowledge financial support from the SNSF. 


\section{REFERENCES}

Allard F., Guillot T., Ludwig H.-G., Hauschildt P. H., Schweitzer A., Alexander D. R., Ferguson J. W., 2003, in Martín E., ed., IAU Symposium, Vol. 211, Brown Dwarfs. Astron. Soc. Pac., San Francisco, p. 325

Amara A., Quanz S. P., Akeret J., 2015, Astron. Comput., 10, 107

Anglada-Escudé G. et al., 2016, Nature, 536, 437

Barnes J. et al., 2014, MNRAS, 439, 3094

Bazot M., Christensen-Dalsgaard J., Gizon L., Benomar O., 2016, MNRAS, 460,1254

Beuzit J.-L. et al., 2008, in McLean I. S., Casali M. M., eds, Proc. SPIE, Vol. 7014, Ground-based and Airborne Instrumentation for Astronomy II. SPIE, Bellingham, p. 701418

Bonavita M., Chauvin G., Desidera S., Gratton R., Janson M., Beuzit J. L., Kasper M., Mordasini C., 2012, A\&A, 537, A67

Claudi R. U. et al., 2008, in McLean I. S., Casali M. M., eds, Proc. SPIE, Vol. 7014, Ground-based and Airborne Instrumentation for Astronomy II. SPIE, Bellingham, p. 70143E

Cutri R. M. et al., 2003, VizieR Online Data Catalog, 2246

Dohlen K. et al., 2008, Proc. SPIE, Vol. 7014, Ground-based and Airborne Instrumentation for Astronomy II. SPIE, Bellingham, p. 70143L

Dupuy T. J., Liu M. C., Ireland M. J., 2011, in Johns-Krull C., Browning M. K., West A. A., eds, ASP Conf. Ser. Vol. 448, 16th Cambridge Workshop on Cool Stars, Stellar Systems, and the Sun. Astron. Soc. Pac., San Fransisco, p. 111

Endl M., Kürster M., 2008, A\&A, 488, 1149

Gilmozzi R., Spyromilio J., 2007, The Messenger, 127, 11

Johns M., 2008, in Andersen T. E., ed., Proc. SPIE, Vol. 6986, Extremely Large Telescopes: Which Wavelengths? Retirement Symposium for Arne Ardeberg. SPIE, Bellingham, p. 698603

Lurie J. C. et al., 2014, AJ, 148, 91

Macintosh B. et al., 2006, Society of Photo-Optical Instrumentation Engineers (SPIE) Conference Series, Vol. 6272, Society of Photo-Optical Instrumentation Engineers (SPIE) Conference Series

Mawet D. et al., 2014, ApJ, 792, 97

Mesa D. et al., 2015, A\&A, 576, A121

Nelson J., Sanders G. H., 2008, in Stepp L. M., Gilmozzi R., eds, Proc. SPIE, Vol. 7012, Ground-based and Airborne Telescopes II. SPIE, Bellingham, p. $70121 \mathrm{~A}$

Sahu K. C., Bond H. E., Anderson J., Dominik M., 2014, ApJ, 782, 89

Soummer R., Pueyo L., Larkin J., 2012, ApJL, 755, L28

Thévenin F., Provost J., Morel P., Berthomieu G., Bouchy F., Carrier F., 2002, A\&A, 392, L9

van Leeuwen F., 2007, A\&A, 474, 653

Vigan A., Moutou C., Langlois M., Allard F., Boccaletti A., Carbillet M., Mouillet D., Smith I., 2010, MNRAS, 407, 71

Vigan A., Gry C., Salter G., Mesa D., Homeier D., Moutou C., Allard F., 2015, MNRAS, 454, 129

Zechmeister M., Kürster M., Endl M., 2009, A\&A, 505, 859

Zurlo A. et al., 2014, A\&A, 572, A85

Zurlo A. et al., 2016, A\&A, 587, A57
${ }^{1}$ INAF - Osservatorio Astronomico di Padova, Vicolo dell'Osservatorio 5, I-35122 Padova, Italy

${ }^{2}$ Núcleo de Astronomía, Facultad de Ingeniería, Universidad Diego Portales, Av. Ejercito 441, Santiago, Chile

${ }^{3}$ Universidad de Chile, Camino el Observatorio, 1515 Santiago, Chile

${ }^{4}$ Aix Marseille Université, CNRS, LAM - Laboratoire d'Astrophysique de Marseille, UMR 7326, F-13388 Marseille, France

${ }^{5}$ European Southern Observatory (ESO), Alonso de Córdova 3107, Vitacura, 19001 Casilla, Santiago, Chile

${ }^{6}$ CRAL, UMR 5574, CNRS, Université Lyon 1, 9 avenue Charles André, F-69561 Saint Genis Laval Cedex, France

${ }^{7}$ Institute for Astronomy, University of Edinburgh, Blackford Hill View, Edinburgh $\mathrm{EH} 9 \mathrm{HHJ}$

${ }^{8}$ INAF - Osservatorio Astrofisico di Arcetri, Largo E. Fermi 5, I-50125 Firenze, Italy

${ }^{9}$ ETH Zurich, Institute for Astronomy, Wolfgang-Pauli-Strasse 27, CH-8093

Zurich, Switzerland

${ }^{10}$ Max-Planck-Institut für Astronomie, Königstuhl 17, D-69117 Heidelberg, Germany

${ }^{11}$ LESIA, Observatoire de Paris-Meudon, CNRS, Université Pierre et Marie Curie, Université Paris Diderot, 5 Place Jules Janssen, F-92195 Meudon,

France

${ }^{12}$ INAF - Osservatorio Astrofisico di Catania, Via S. Sofia 78, I-95123 Catania, Italy

${ }^{13}$ INAF - Osservatorio Astronomico di Capodimonte, Via Moiariello 16, I-80131 Napoli, Italy

${ }^{14}$ Université Grenoble Alpes, IPAG, F-38000 Grenoble, France

${ }^{15}$ CNRS, IPAG, F-38000 Grenoble, France

${ }^{16}$ INAF - Istituto di Astrofisica Spaziale e Fisica Cosmica di Milano, Via E. Bassini 15, I-20133 Milano, Italy

${ }^{17}$ Department of Astronomy, Stockholm University, SE-10691 Stockholm, Sweden

${ }^{18}$ Institute of Astronomy, University of Cambridge, Madingley Road, Cambridge $\mathrm{CB} 3 \mathrm{OHA}$

${ }^{19}$ Laboratoire Lagrange (UMR 7293), UNSA, CNRS, Observatoire de la Côte d'Azur, Bd. de l'Observatoire, 06304 Nice Cedex 4, France

${ }^{20}$ Department of Astronomy, University of Michigan, Ann Arbor, MI 48109

${ }^{21}$ Observatoire de Genéve, University of Geneva, 51 Chemin des Maillettes, 1290 Versoix, Switzerland

${ }^{22}$ NASA Exoplanet Science Institute, California Institute of Technology, Pasadena, CA, USA

${ }^{23}$ Dipartimento di Fisica e Astronomia 'G. Galilei', Universitá degli Studi di Padova, Vicolo dell'Osservatorio 3, I-35122 Padova, Italy

This paper has been typeset from a $\mathrm{T}_{\mathrm{E}} \mathrm{X} / \mathrm{L} \mathrm{T} \mathrm{E} \mathrm{X}$ file prepared by the author. 